\title{
Using Analytics To Challenge Conventional Baseball Wisdom
}

\author{
Loren Paul Rees, Virginia Tech, USA \\ Terry R. Rakes, Virginia Tech, USA \\ Jason K. Deane, Virginia Tech, USA
}

\begin{abstract}
Baseball, like most other sports, has a set of tenets that began early and have survived virtually unquestioned. Modern analytics gives us an opportunity to examine some of these long-held tenets to see if they were founded on solid evidence. This research examines some common baseball wisdom through an initial study utilizing simulation. In particular, the profiles of several baseball teams are constructed and various factors are examined by simulating ten baseball seasons under various configurations with the different teams.

Contrary to conventional wisdom, a batting order where high-average hitters bat third in a lineup and the team's best power hitter bats cleanup (fourth), for example, does not necessarily generate the most runs per game over the long run. Moreover, high-average hitters with less power can generate more runs per game than power hitters with lesser averages. Finally, it appears that hitters who perform well with runners in scoring position are more influential in helping their team score more runs than even more powerful or higher average hitters who do not produce as frequently in such cases. Players with lower star profiles, but who rise to the occasion with runners in scoring position, can often be purchased by baseball clubs that have a more constrained payroll; teams that are less well-off financially may thus purchase or trade for these hitters and still field a team with a competitive level of high run production.
\end{abstract}

Keywords: Analytics; Baseball; Simulation

\section{INTRODUCTION}

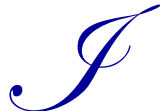

$\mathrm{n}$ the mid-1970s, Bill James, a night watchman at a bean cannery, began analyzing baseball box scores, without a computer, with the purpose of questioning baseball's long-held presuppositions, or "baseball wisdom" as it is sometimes called (Hanchett, 2014). James' analytical musings have now evolved into a whole field - sports analytics. There is not complete agreement as to what the term means now or what it includes and excludes [one definition of sports analytics is "the utilization of technology to answer questions [about sports] correctly that the average human mind cannot without bias or error" (Bessire, 2014)]. However, the impact of analytics is clear as league luminaries of multiple sports, front-office decision-makers, and industry leaders are employing analytics in decision-making. Moreover, there are now meetings and conferences with research papers and panelists discussing the increasing use of analytics on the field, in the front office, and in the boardroom. Probably the preeminent sports analytics conference is the MIT Sloan Sports Analytics Conference, where topics discussed this year included American football, baseball, basketball, and golf (Wikipedia, 2014c). There is now a journal exclusively devoted to sports analytics - "The Journal of Sports Analytics (JSA)" - which aims to be a forum for the discussion of practical applications of sports analytics research, serving primarily team owners, general managers, and coaches. (JSA, 2014). Moreover, other excellent journals without a sports emphasis have been publishing research on sports analytics, including this journal (Bartholomew and Collier, 2011). Topics are widely diverging in these journals, ranging from playing basketball ("Every basketball player takes and makes a unique spatial array of shots. In recent years, technology to measure the coordinates of these constellations has made analysis of them possible" (Shortridge, Goldsberry, Adams, 2014) to refereeing Italian football matches ("Empirical research allows for the verification of the incidence of systematic errors on the decision-making processes of 
referees" (Lombardi, Trequattrini, and Battista, 2014)), and now yet another term has entered the analytics lexicon sabermetrics - which is the term for the empirical analysis of baseball, especially baseball statistics that measure ingame activity. The term is derived from the acronym SABR which stands for the Society for American Baseball Research (Wikipedia, 2014d). The research presented in this paper is based on in-game statistics, but instead of doing statistical analyses on those data, those statistics are used to perform computer simulations of baseball games.

Major League Baseball (MLB) - America's so-called national pastime - is one of four major professional sports leagues in North America. There are 30 teams in MLB, with 29 from the United States and one from Canada. MLB has the highest season attendance of any sports league in the world with more than 74 million spectators in 2013 (Wikipedia, 2014b).

The amount of financial resources, or player payroll available to the individual teams, varies dramatically across the league, with teams such as the New York Yankees having a much larger budget than many of their competitors (Wikipedia, 2014a). It is not surprising that those with more significant resources are often able to attain higher levels of on-the-field achievement given their ability to outbid their competitors in an effort to procure the most sought-after players. The question arises as to whether analytics can be used to identify and then obtain players who are not known stars and who do not necessarily have the best offensive statistics, e.g., do not have the most home runs or the highest batting averages. This question was the foundation of the recent film Moneyball, where regression and econometrics were used for scouting and analyzing players, allowing the Oakland Athletics to win twenty consecutive games - an American League record. In this research, the authors use computer simulation for performing similar analytics.

\section{Purpose and Plan of Presentation}

The scope of this paper is to examine the hitting aspect of baseball in order to develop insight into 1) the best types of players to acquire and 2) how to arrange them in batting order. This information should be useful to both on-the-field managers who decide which team players to play any given day and in what order they should bat, as well as for general managers who are trying to acquire (purchase, draft, and/or trade for) the best set of players that the franchise's budget allows.

The purpose of this paper is 1) to examine the effect of hitters who hit differently with runners in scoring position (with runners on second and/or third base), 2) to study different types of batting lineups (orders in which players bat), 3) to ascertain the relative advantage of players who are power hitters, as opposed to those who have less power but hit with a higher batting average, and 4) the relative benefit in terms of runs scored per game as a function of different batting averages.

The next section provides details of the methodology, including the four key components of baseball wisdom to be investigated, and subsequent sections present results for each of these factors, a discussion of the results, and conclusions.

\section{METHODOLOGY}

The simulation model examines only the offensive portion of baseball; that is, only that portion of major league baseball when a team is at bat. While it is true that certain pitchers dominate hitters, whereas others are not as effective, pitching is ignored as a control factor because the simulation produces average statistics, as a player's average performance is measured against all teams and all pitchers in his or her league. Also, issues, such as double plays, sacrifice flies, and infield fly rules, were ignored in this study but could be included in a more detailed study.

\section{Simulation Model}

Three main portions of the simulation model are shown schematically in Figure 1: 1) how an "at bat" is modeled, 2) how outs are processed, and 3) how runners are moved around the bases (with a three-base hit used as an illustration). The simulation is written in Arena version 14.0 (Rockwell) and the schematics shown in Figure 1 are from the Arena language. The basic concept is that batters get hits a certain percentage of the time based on 
their batting average and when they do get a hit, there is a certain probability that the hit is a one-base hit (single), two-base hit (double), three-base hit (triple), or a four-base hit (home run).

Figure 1a. Modeling an At Bat

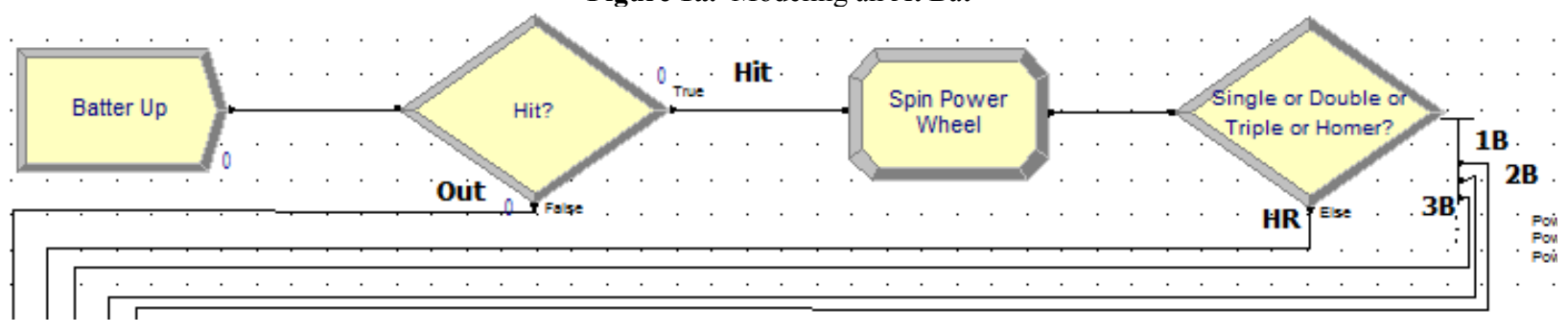

Figure 1b. Modeling an Out

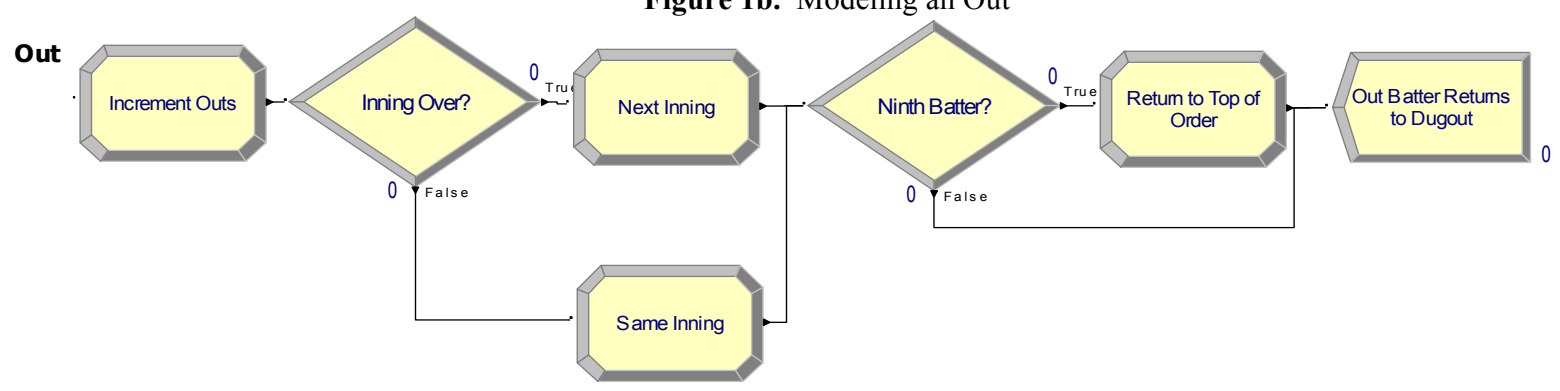

Figure 1c. Modeling A Triple (A Three-Base Hit)

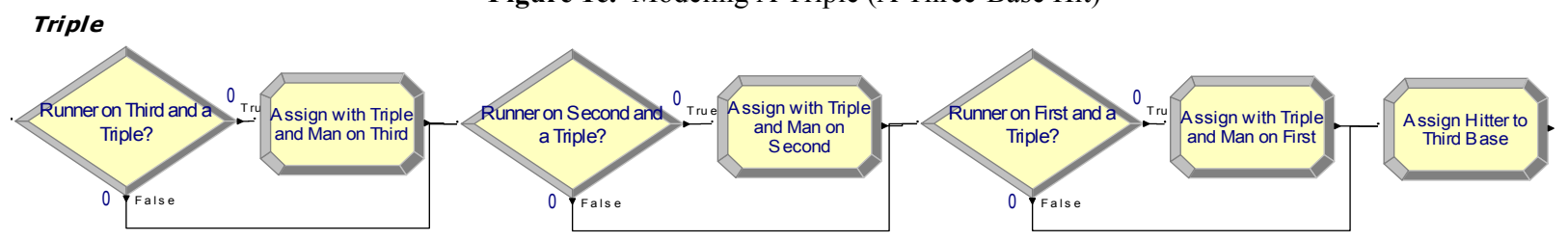

Batting averages are included in the model by sampling from a simple discrete distribution. For example, consider a player who is hitting 0.300 , which means the player gets a hit thirty percent of the time. A random number is drawn and if the number is less than or equal to 0.300 , the batter got a hit. In Figure 1a, this procedure is implemented in the diamond-shaped box labelled "Hit?". If a number greater than the hitter's batting average is drawn, the batter has made an out. The manner in which outs are processed is shown in Figure 1b. If the batter gets a hit, the batter moves on to the "Spin Power Wheel" block in Figure 1a, where the conditional percentages for this particular batter getting a single/double/triple/homer/ (e.g., 75/20/2/3/), given he has a hit, are looked up. Another random number is used to determine the type of hit. The batter then branches to the section of code for the given hit.

For instance, if the batter gets a three-base hit, the process shown in Figure 1c is followed. First, the model checks to see if there already is a runner on third base. If not, the next block - an Arena 'Assign' block labeled 'Assign with Triple and Man on Third' - is skipped. If there was a man on third already, then the "'Assign' block is executed which 1) records that a run has been scored and 2) removes the runner who just scored from third. Next, there is a check to see if there was a runner on second base when the triple was hit. If so, that runner is scored and removed from second base. Next, a check is made to see if there was a runner on first base and, if so, that runner is scored and removed from the base paths. Finally, regardless of whether there were any runners on base before the triple, the batter is placed on third base and the model passes control to ensure that the next batter is brought to the plate to hit. Similar code is written for the cases where batters hit singles, doubles, and homers. 


\section{Experimental Factors}

The underlying motivation of this paper is to provide an initial investigation of several common baseball strategies. Factors are chosen which enable analysis of these strategies. Table 1 is a list of the four factors and associated levels.

Table 1. Factors Investigated in Study

\begin{tabular}{|c|c|c|c|c|c|c|}
\hline$\#$ & FACTOR & \#LEVELS & \multicolumn{4}{|c|}{ LEVELS } \\
\hline 1 & Scoring Position (add \% to BA) & 3 & \multicolumn{4}{|c|}{$-0.075 ; 0.000 ;+0.075$} \\
\hline 2 & $\begin{array}{l}\text { Type Lineup } \\
\text { traditional } \\
\text { all batters have same BA } \\
\text { descending batting average } \\
\text { random batting order }\end{array}$ & 4 & \multicolumn{4}{|c|}{$\begin{array}{c}\text { e.g., } 0.300,0.275,0.325, \ldots \\
\text { e.g., } 0.300,0.300,0.300, \ldots \\
\text { e.g., } 0.325,0.300,0.275, \ldots \\
\text { e.g., } 6,4,5,2,3,8,1,7,9\end{array}$} \\
\hline 3 & $\begin{array}{l}\text { Power profile (\% 2Bs; \% HRs) } \\
\text { Singles Hitters } \\
\text { Power Hitters, lesser } \\
\text { Power Hitters, more so }\end{array}$ & 3 & $\begin{array}{l}\mathbf{1 B} \\
75 \\
66 \\
56\end{array}$ & $\begin{array}{l}\mathbf{2 B} \\
15 \\
20 \\
24\end{array}$ & $\begin{array}{c}3 \mathbf{B} \\
5 \\
2 \\
3\end{array}$ & $\begin{array}{c}\text { HR } \\
5 \\
12 \\
17\end{array}$ \\
\hline 4 & Team Batting Average & 3 & \multicolumn{4}{|c|}{$0.225 ; 0.250 ; 0.275$} \\
\hline
\end{tabular}

Factor 1: Hitting With Runners In Scoring Position

Major league baseball teams all desire players who have high batting averages and who also hit for power. Unfortunately, such players demand large salaries and, therefore, baseball teams with relatively limited financial resources cannot afford many of these players. The difficulty of this challenge is exacerbated by the presence of competitors, such as the Boston Red Sox and the New York Yankees, who are not as constrained financially. Thus, financially poorer teams are constantly looking for ways to identify and acquire players who are less expensive, yet still allow them to field a high scoring team.

One possible strategy is for teams to purchase/utilize players who hit particularly well when runners are in scoring position; i.e., when runners are either on second base, third base, or both. The term "scoring position" is used in baseball parlance because, generally speaking, a hit of any kind will score the base runner.

Note that what is being investigated here differs from the term "clutch hitter" - one who produces when the pressure is on in late innings of the game, in critical games during the pennant race or World Series, hitting with runners in scoring position when there are two outs, etc. (Getz, 2013). This model looks at what a hitter does whenever runners are in scoring position, whether this situation occurs early in the game, regardless of how many outs there are, or even in a game that the media and fans do not deem critical.

The authors are not the first to suggest that an ability to drive home runners in scoring position is critical. An article in May 2010 made the point. "As the 2010 Major League Baseball season transitions from April showers to May flowers, one issue is abundantly clear - Winning, by and large, is directly tied to a team's ability to drive home runners in scoring position (RISP).

"It shouldn't be surprising that two of the top three teams in that category - Tampa Bay and San Diego entered the weekend in first place. The third - Arizona - also has had a productive first month and was only 1-1/2 games behind the Padres in the National League West. "Conversely, the four teams struggling most to knock home runners once they get into scoring position are having problems winning. Entering the weekend, Baltimore (with a RISP of 0.193) was 4-18 and had the worse record in either league.

In this work, the RISP factor is modeled by introducing three potential levels of batting average for a given player if at least one runner is in scoring position. The three levels result in 1) adding 75 points to the batter's normal batting average, 2) leaving the batting average as it is - this hitter hits the same whether runners are in scoring position or not, and 3) the hitter (perhaps feeling some pressure) actually decreases his percentage of getting 
a hit by 75 points. The hitter's underlying batting average for the simulation is restored to its original value after each RISP at bat. In other words, with RISP the hitter's batting average is adjusted for just this one at bat.

Factor 2: Type of Lineup

Baseball lore and wisdom decree that a particular arrangement of hitters be utilized by a manager in a season, with high-average hitters batting third and a power hitter batting cleanup (fourth), for example; but is this the arrangement most likely to guarantee maximal run-scoring potential over the course of a season?

To address the question, four different lineup orders are examined: 1) in the first lineup, the batters hit in a traditional order; 2) in the second lineup,- one of perhaps theoretical interest only, all batters are given the same batting average; namely, the team average; 3 ) in the third lineup, batters are arranged in a descending batting average order, ostensibly because the batters with the best averages should bat most frequently in a game; and 4) in the fourth lineup, batters are sent to bat in a totally random order in every game they play.

A similar analysis from the mid-1970s, using a programmed embodiment of the Sports Illustrated baseball game available at that time, appeared in Operations Research (Freeze, 1974). Freeze's analysis had two teams playing each other and his metric was number of wins per 162-game baseball season. This analysis differs in that the statistics are almost forty years more current but also in that the metric is runs scored per game; the possible confounding performance of the opposing team is removed. Freeze discovered that the effect of the most successful lineup versus the least successful was less than three wins per season.

\section{Factor 3: Power Profiles}

To address the question of what type of player a baseball team should trade for (a high-average singles hitter or a lower-average slugger), the model uses three different power profiles. The first profile has hitters that are primarily singles hitters, having a percentage distribution of types of hits of $75 / 15 / 5 / 5$. Note that the percentages for both doubles and homeruns were specified as shown because they were the percentage of doubles and homers for the team in the majors with the least number of these types of hits. The second profile has a batting order with moderately powerful hitters, with a percentage profile of $66 / 20 / 2 / 12$. These percentages were for the team with the average number of doubles and homeruns in 2012. Finally, a more powerful profile of power hitters was used with percentages of hits distributed 56/24/3/17. The team with the highest percentage of doubles and home runs in the majors in 2012 had these percentages. In short, the percentages chosen as levels for this factor are based on major league baseball 2012 statistics.

\section{Factor 4: Team Batting Average}

Finally, the effect of team batting average (BA) on runs per game was examined. It is obvious that players with higher batting averages are better run producers than those with lower averages, but how significant is batting average when compared with other factors? Is a 25-point increase in BA, when a team is hitting 0.225, more valuable than a similar increase to a team already hitting for a higher percentage, such as 0.250 ?

The major league baseball team with the best organizational batting average in 2012 batted 0.276 . On the other end of the spectrum, the team with the worst average batted 0.233 and the league-wide team batting average was 0.253 . Consequently, the three levels with respect to this factor are 1) 0.225 ,2) 0.250 , and 3) 0.275 .

\section{RESULTS}

\section{Hitting with Runners in Scoring Position}

If a General Manager is trying to build a team with limited resources, or an on-the-field manager is trying to maximize run production with an existing set of players, simulation results indicate that he or she should consider a hitter's productivity with runners in scoring position as a primary decision input. In particular, for a team with an overall batting average of 0.250 , an increase of 60 points in batting average with RISP will result in one additional 
run per game. Conversely, a drop in 68 points in batting average with RISP (i.e., "choking") will cost the team one run per game (see Figure 2). Stated differently, if a General Manager can only afford 0.250 hitters and (s)he has a choice in purchasing/trading for one set hitting 60 points higher (i.e., .310) with runners in scoring position and a group hitting no differently (0.250) when runners are in scoring position, choosing the former set will result in an extra run per game. The primary take away is that this strategy of run production improvement is likely to be considerably less costly than obtaining a similar increase by obtaining a set of hitters with the overall improvement necessary in overall batting average to produce an extra run. See the discussion below on factor 4 - team batting average.

Figure 2. Runs Per Game As A Function Of An Increase/Decrease In Batting Average With Runners In Scoring Position

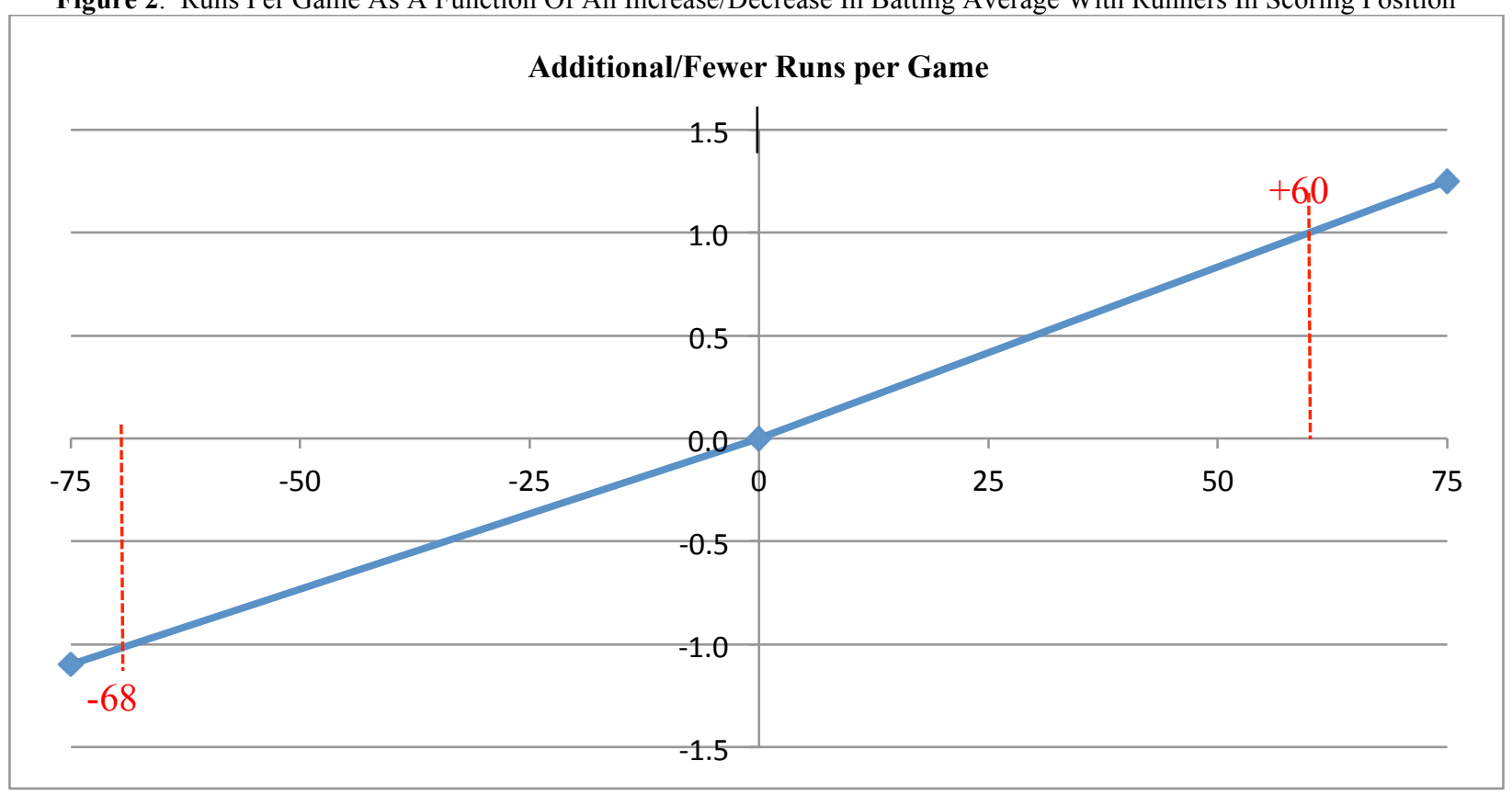

While it is certain that general managers and on-the-field managers have a general sense of how players tend to perform in critical situations, it is much less likely that they have detailed statistics on a given player's ability to hit with runners in scoring position. To highlight a key point of these findings, consider a simple question. If you were manager for a day and had the opportunity and resources to insert Reggie Jackson into your lineup, would you? Like most managers, you would probably jump at the opportunity; after all, he is "Mr. October". Based on the findings in this research, this might not be a good move for your team. Baseball fans and journalists nicknamed Reggie Jackson "Mr. October" because he performed exceptionally well in the World Series; however, a close analysis of Reggie's performance statistics clearly show that he hit much worse with runners in scoring position in the other months of the baseball season (Appling, 2006), meaning that he would likely not be your best use of resources in an effort to achieve season-long success. From the model, it is clear that ability to hit with RISP is an important statistic that should be collected and used. The expansion of analytics into baseball and the routine collection and analysis of in-game data should make this an easy task.

\section{Type of Lineup}

As mentioned earlier, four different batting lineup strategies were considered in this analysis. The type of lineups included: 1) the traditional lineup, 2) a hypothetical lineup whereby every player on the team has exactly the same batting average (note that in all four cases, the team-average batting averages are exactly the same), 3) a descending batting average order whereby players are ordered from the player with the highest batting average hitting first, down to the player with the worst average (usually the pitcher) hitting ninth, and 4) a totally random batting average whereby the order the players hit is drawn at random for each game. 
Results for the four scenarios are shown in Figure 3 and are quite contrary to conventional baseball wisdom. The traditional baseball lineup did worst scoring the fewest runs, while the random order performed best and the hypothetical all-hitters-with-the-same-average case scored second best. These results suggest that managers making traditional lineups may actually be costing themselves a run every six or seven games $(4.55-4.40=0.15$ runs per game) by not making daily lineups randomly.

Figure 3. Runs Per Game Versus Type of Lineup

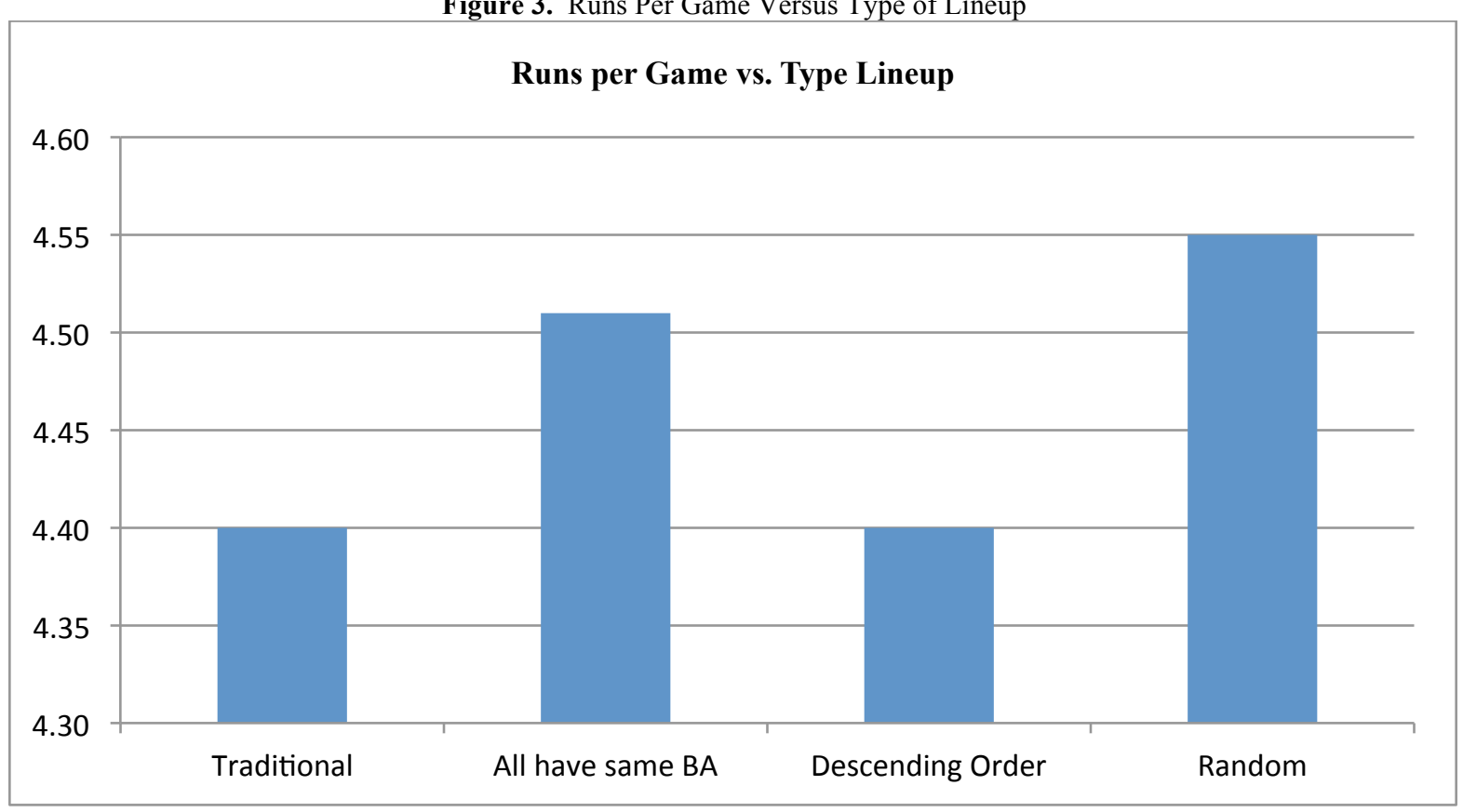

\section{Power Profiles}

Going back to the 1959 Chicago White Sox, people have debated the relative importance of the power versus batting average profile of players and teams. Generally, power hitters are paid better than singles hitters.

In order to investigate this tradeoff, the three scenarios discussed earlier were examined. Results (see Table 2) are in the form of a tradeoff matrix, showing the effect of 25 points in batting average between adjacent columns and the three power profiles. Note, for example, that a team with all singles hitters batting 0.250 scores almost as many runs per game (4.05) as a team with all heavy power hitters (4.08), but batting only 0.225 . Similarly, a team with all singles hitter batting 0.275 scores slightly more runs per game (4.98) as a team with all heavy power hitters (4.97), but batting 0.250 . It is quite possible that, examining these results from a general manager's point of view, the singles-hitting team would be much less expensive (and it would also be possible that singles hitters would be faster and better defensive players as well - factors not examined in this paper).

Table 2. The Tradeoff Matrix for Batting Average Versus Power

\begin{tabular}{lccc}
\hline & $\begin{array}{c}\text { Hitters w/ 0.225 BA } \\
\text { (F1=1) }\end{array}$ & $\begin{array}{c}\text { Hitters w/ 0.250 BA } \\
\text { (F1=2) }\end{array}$ & $\begin{array}{c}\text { Hitters w/ 0.275 BA } \\
\text { (F1=3) }\end{array}$ \\
\hline Singles Hitters (F2=1) & 3.34 & 4.05 & 4.98 \\
Medium-Power Hitters (F2=2) & 3.42 & 4.23 & 5.16 \\
Heavy-Power Hitters (F2=3) & 4.08 & 4.97 & 5.94 \\
\hline
\end{tabular}

Note: All cases shown are for (1) hitters with no change in average with runners in scoring position (factor $3=2$ ), (2) hitters batting in the traditional lineup (factor $4=1$ ), and (3) the three rows (factor 2) have power profiles defined as follows:

$\mathrm{F} 2=1: 75 / 15 / 5 / 5, \mathrm{~F} 2=2: 66 / 20 / 2 / 12$, and $\mathrm{F} 2=3: 56 / 24 / 3 / 17$, where $\mathrm{a} / \mathrm{b} / \mathrm{c} / \mathrm{d}$ represent 'a' percent singles, ' $\mathrm{b}$ ' percent doubles, 'c' percent triples, and 'd' percent homeruns. 


\section{Team Batting Average}

Looking at Figure 4, it is obvious, and not surprising, that an increase in team batting average leads to an increase in run generation. However, two points should be noted: 1) slope is not constant and 2) it can be more expensive for a general manager to get an extra run per game by buying additional batting average than to get the extra run through purchasing a hitter who hits well with runners in scoring position.

Figure 4. Runs Per Game Versus Team Batting Average

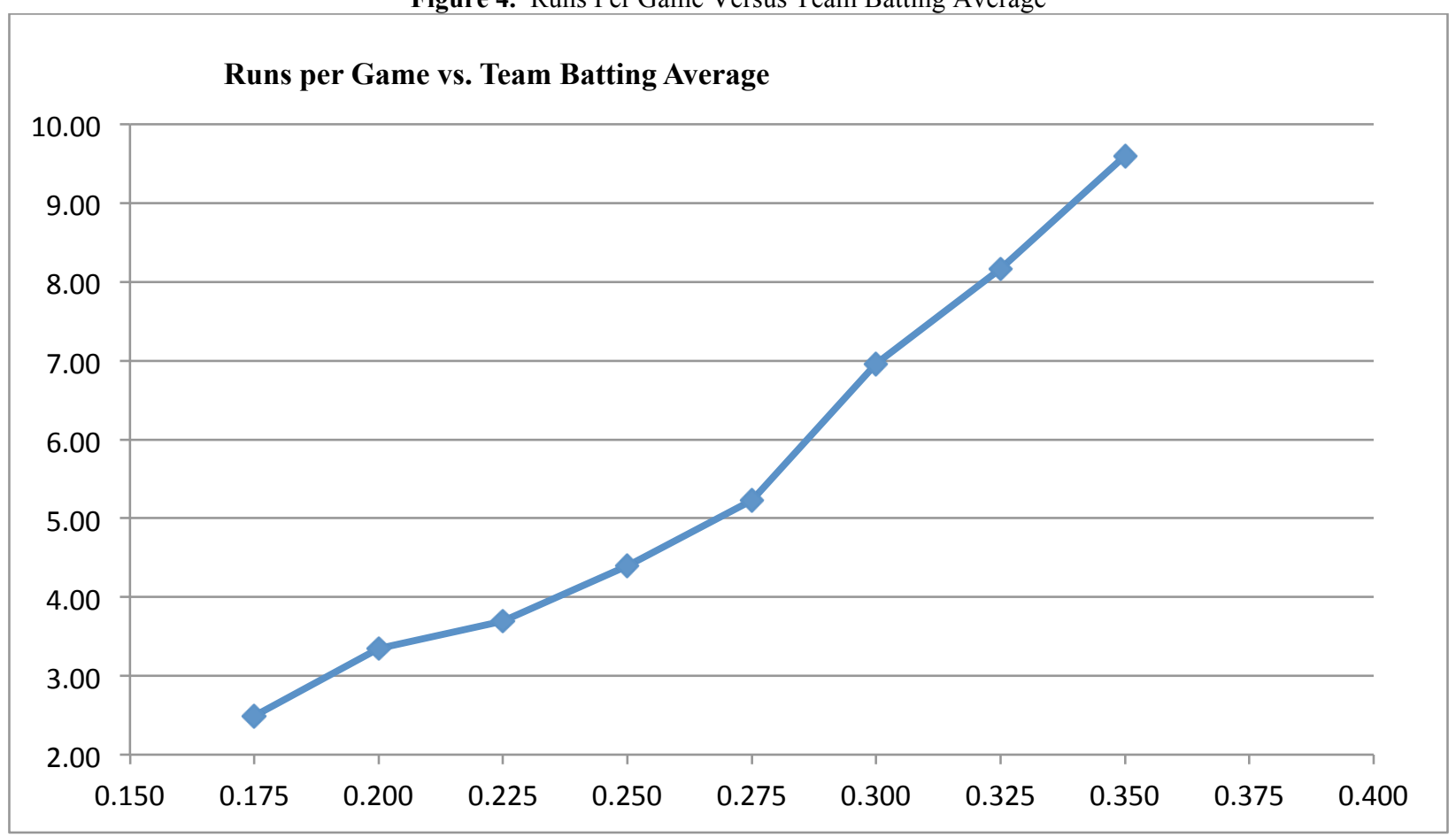

With regard to the non-constant slope, note that starting with a team hitting 0.250 and increasing the team's overall average to 0.275 will result in an additional 0.8 runs per game, whereas starting with a team with a 0.275 average and increasing that team's average by 25 points will lead to approximately 1.9 additional runs per nine innings. Conversely, losing 25 points of team batting average through age, injury, or trading down for financial reasons will impact a team differently depending on the team's batting average before the decline.

If a general manager has an existing team with an overall batting average of $\sim 0.230$, then to get an additional run per game through batting average, she or he will have to buy players with an average of 35-40 points better in batting average. This could be fairly costly. Alternatively, to get an extra run per game through purchasing hitters who hit well with runners in scoring position, the GM will still be able to purchase players with the same batting average but with a 60-point increase in batting average with runners positioned to score. Such a strategy could be considerably cheaper, particularly if other GMs, the media, and fans are unaware of players' performance in this regard or are unsure of its importance.

\section{CONCLUSIONS}

This research examines several aspects of conventional baseball wisdom and shows that several appear to be in question. It demonstrates that computer simulation analysis can provide insight, both with respect to the particular team profile a team should attempt to achieve and to how and when those players should be played, at least from an offensive standpoint. The study examines the effects of several components of a hitter's portfolio; namely, the importance of his hitting with runners in scoring position, where he bats in the lineup and the importance of his ability to hit for average versus his ability to hit with power. It appears that the answers to these 
questions are not obvious, even to those in MLB, yet the answers to these questions are obtainable with computer simulation analysis. Simulation is a valuable and inexpensive tool that should at least be investigated in depth with detailed information for teams with interest in increasing run productivity. A Decision Support System (DSS) built around extensive situational batting data and sound analytics modeling could provide a definite advantage in recruiting and scheduling. The generation of extensive data for a team representing the additional detailed information on its players that has been outlined here (such as BA with RISP) should provide further specific insight into increased run production capabilities. Baseball teams would do well to develop an individual team DSS to examine trading implications and lineup issues for the players and to exploit the competitiveness the team presently possesses.

\section{AUTHOR INFORMATION}

Loren Paul Rees is Andersen Professor of Business Information Technology. He received the Ph.D. in Industrial and Systems Engineering, B.E.E. from Georgia Tech, and M.S.E.E. from the Polytechnic Institute of Brooklyn. His current research focuses on multi-hazard disaster analysis and managerial issues in information security. He has published in Naval Research Logistics, IIE Transactions, Decision Sciences, Transportation Research, Journal of American Medical Informatics Association, Journal of the Operational Research Society, Computers and Operations Research, Communications of the ACM, Decision Support Systems, and others. Email: loren@vt.edu.

Terry R. Rakes is William and Alix Houchens Professor of Information Technology at Virginia Tech. He received the Ph.D. in Management Science, M.B.A. and B.S.I.E. from Virginia Tech. His research interests are in analytics and big data analysis, text and data mining, disaster planning and logistics, and information security. He has published in Management Science, Decision Sciences, Decision Support Systems, Annals of Operations Research, OMEGA, European Journal of OR, Operations Research Letters, Information and Management, Journal of Information Science, and others. Email: trakes@vt.edu.

Jason K. Deane is Associate Professor of Business Information Technology in the Pamplin College of Business at Virginia Tech. He received a Ph.D. in Decision and Information Sciences from the University of Florida and an M.B.A. and B.S. in Business Administration from Virginia Tech. His current research interests are in the areas of artificial intelligence, computer-aided decision support systems, information system security, large scale optimization and information retrieval. He has published in such journals as Decision Support Systems, Annals of Operations Research, Information Technology and Management, International Journal of Physical Distribution and Logistics Management, Operations Management Research, and others. Email: jdeane1@vt.edu.

\section{REFERENCES}

Appling. (2006). "Baseball Fever,” Retrieved from http://baseball-fever.com/showthread.php?38738-Hitting-withRISP.

Bartholomew, J. T., \& Collier, David A. (2011). “The Role of Contested and Uncontested passes in Evaluating Defensive Basketball Efficiency," Journal of Service Science, 4(2) 33-42.

Bessire, Paul. (2014). "Analytics!(?)," Retrieved from http://www.predictionmachine.com/analtyics-sportsconference-bill-james.

Freeze, R. Allen. (1974). "An Analysis of Baseball Batting Order by Monte Carlo Simulation, Operations Research, 22(4) 728-735.

Getz, Vin. (2013). "Derek Jeter and the 20 Most Clutch Hitters of the Past 60 Years (Most RBI with 2 Outs and RISP).” Retrieved from http://sportslistoftheday.com/2013/08/03/derek-jeter-and-the-20-most-clutchhitters-of-past-60-years-most-rbi-with-2-outs-and-risp/.

Hanchett, Doug. (2014). "Playing Hardball with Big Data: How Analytics is Changing the World of Sports". Retrieved from http://www.emc.com/collateral/article/137534-sports-analysis.pdf.

JSA. (2014). "Journal of Sports Analytics". Retrieved from http://journalofsportsanalytics.com/.

Lombardi, Rosa, Trequattrini, Raffaele, \& Battista, Mirella. (2014). "Systematic errors in decision making processes: the case of the Italian Serie A football championship," Int. J. of Applied Decision Sciences, 7(4) 239-254.

Rockwell Automation. (2013). Arena Simulation Software, version 14.0. Milwaukee, WI. 
Shortridge, Ashton, Goldsberry, Kirk, Adams, \& Matthew. (2014). "Creating space to shoot: quantifying spatial relative field goal efficiency in basketball," Journal of Quantitative Analysis in Sports, 10(3) 303-313.

Wikipedia. (2014a). "List of highest paid Major League Baseball players". Retrieved from http://en.wikipedia.org/wiki/List of highest paid Major League Baseball players.

Wikipedia. (2014b). "Major League Baseball," Retrieved from http://en.wikipedia.org/wiki/Major League Baseball.

Wikipedia (2014c). "MIT Sloan Sports Analytics Conference". Retrieved from http://en.wikipedia.org/wiki/MIT Sloan Sports Analytics Conference.

Wikipedia. (2014d). "Sabermetrics," Retrieved from http://en.wikipedia.org/wiki/Sabermetrics. 